\title{
Image of the month: Rapidly evolving mitral stenosis: an unusual case of endocarditis
}

\author{
Authors: David McBriar, ${ }^{A}$ Aileen Kearney ${ }^{B}$ and Ben Cole ${ }^{C}$
}

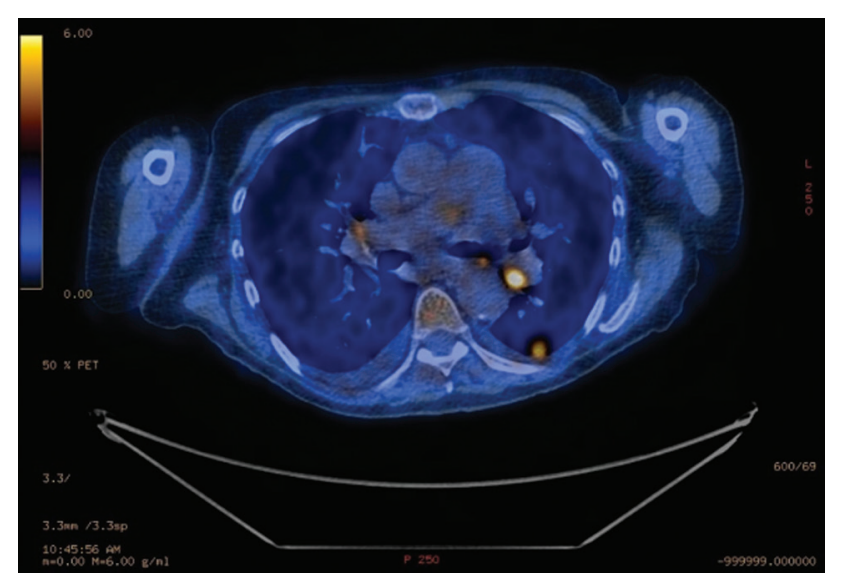

Fig 1. Positron emission tomography - computed tomography showing the primary sub-pleural nodule and mediastinal lymph nodes.

KEYWORDS: Marantic, endocarditis, mitral, stenosis

DOI: $10.7861 /$ clinmed.2020-0060

\section{Case presentation}

A 72-year-old woman was admitted with increasing dyspnoea. She had a background of mitral regurgitation, atrial fibrillation (for which she was anticoagulated with apixaban), chronic obstructive pulmonary disease and primary hyperthyroidism. Computed tomography revealed an $11 \mathrm{~mm}$ sub-pleural nodule in the left lower lobe. Positron emission tomography indicated fluorodeoxyglucose avidity in the nodule, with further hilar nodal uptake (Fig 1). Transbronchial fine needle aspiration of hilar nodes

Authors: ${ }^{\text {A }}$ cardiology senior house officer, Mater Infirmorum, Belfast, UK; ${ }^{B}$ Cardiology registrar, Mater Infirmorum, Belfast, UK; ${ }^{C}$ consultant cardiologist, Mater Infirmorum, Belfast, UK confirmed a diagnosis of squamous cell carcinoma. Transthoracic echocardiography demonstrated preserved left ventricular systolic function, moderate to severe mitral regurgitation and moderate mitral stenosis. Her case was discussed at the respiratory multidisciplinary team meeting and it was felt that she would not be fit for active treatment.

Five months later, she represented with worsening dyspnoea and peripheral oedema. Repeat transthoracic echocardiography demonstrated thickened mitral valve leaflets causing severe stenosis, and severe right ventricular systolic dysfunction. Subsequent transoesophageal echocardiography revealed a large mass extending between the leaflets causing severe mitral stenosis (Fig 2).

Based on imaging findings, the absence of clinical signs of infection, sterile blood cultures and negative lupus serology; a diagnosis of non-bacterial thrombotic endocarditis was made. Her anticoagulant was switched to warfarin, and she responded well to diuretics. Two months following discharge, she remained stable.

\section{Discussion}

Non-bacterial thrombotic endocarditis (NBTE) describes sterile platelet thrombi deposition on heart valves. It was formerly known as marantic endocarditis as it was often encountered in critically unwell patients, or at autopsy. ${ }^{1}$ NBTE is rare and shows no sex predilection. It is primarily associated with cancer, usually adenocarcinoma. It has also been described in association with rheumatoid arthritis, sepsis and burns.'

Pulmonary and cerebral emboli are the primary presenting features of NBTE, as well as embolisation of the spleen, kidney and skin. ${ }^{2}$ Presentation with valvular dysfunction, as described here, is less common.

Treatment of NBTE is directed at the underlying disease, however this is often impossible owing to late presentation. Surgical debridement is rarely performed. ${ }^{3}$ As there is little inflammatory reaction at the site of endocarditis, systemic embolisation is more common than infective endocarditis and so all patients should be anticoagulated. Heparin is the recommend anticoagulant, although warfarin may be used in patients with longer life expectancy. ${ }^{4}$ 

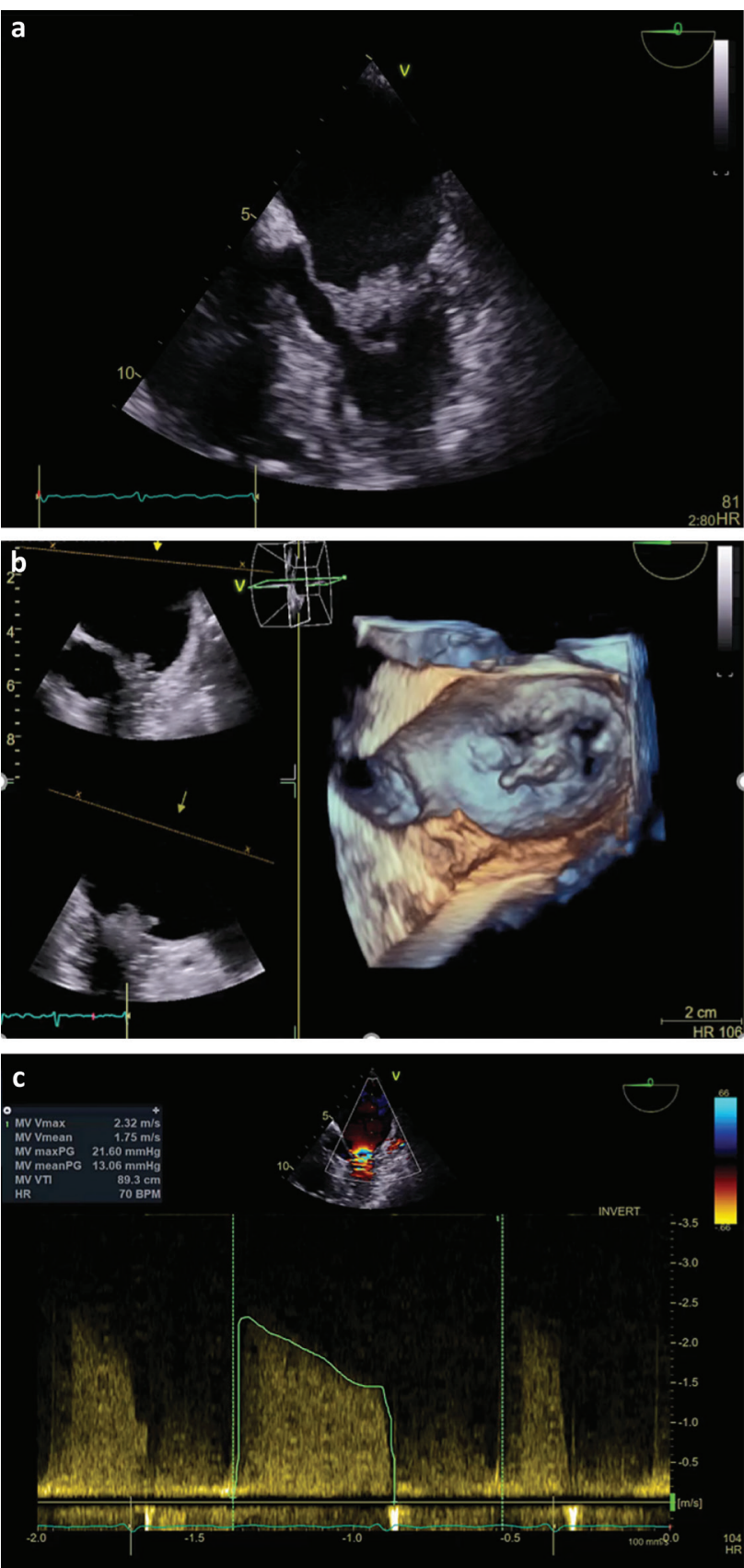

Fig 2. a) 2D still image of mitral valve. b) 3D still image of mitral valve. c) Continuous wave Doppler through mitral valve; peak gradient of $21.6 \mathrm{mmHg}$ and mean gradient of $13 \mathrm{mmHg}$ in keeping with severe mitral stenosis.

\section{References}

1 Liu J, Frishman WH. Nonbacterial thrombotic endocarditis. Cardiol Rev 2016;24:244-7.

2 El-Shami K, Griffiths E, Streiff M. Nonbacterial thrombotic endocarditis in cancer patients: pathogenesis, diagnosis, and treatment. Oncologist 2007;12:518.

3 Rabinstein AA, Giovanelli C, Romano JG et al. Surgical treatment of nonbacterial thrombotic endocarditis presenting with stroke. J Neurol 2005:252:352-5.

4 Bell WR, Starksen NF, Tong S et al. Trousseau's syndrome. Devastating coagulopathy in the absence of heparin. Am J Med 1985;79:423-30.

Address for correspondence: Dr David McBriar, Mater Infirmorum, Crumlin Road, Belfast BT14 6AB, UK. Email: david.mcbriar@outlook.com 


\section{Prolonged disorders of consciousness following sudden onset brain injury National clinical guidelines}

New guidelines from the Royal College of Physicians, endorsed or supported by a further 15 health bodies, offer updated guidance on the diagnosis, assessment, care and management of patients with prolonged disorders of consciousness.

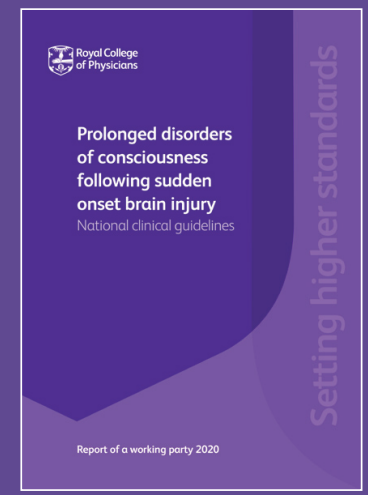

Download the updated guidelines www.rcplondon.ac.uk/pdoc 